\title{
A FIXED POINT THEOREM FOR A SYSTEM OF MULTIVALUED TRANSFORMATIONS
}

\author{
S. CZERWIK
}

\begin{abstract}
We shall prove a fixed point theorem for a system of multivalued mappings which generalizes the result obtained by the author [1, Theorem 1]. For $n=1$ we obtain a generalization of results of Reich [5, Theorem 5] and Nadler [3, Theorem 5], [4, Theorem 1].
\end{abstract}

1. Let $(X, d)$ be a metric space. We follow the notation of [4].

(a) $C L(X)=\{C: C$ is a nonempty closed subset of $X\}$,

(b) $N(\varepsilon, C)=\{x \in X: d(x, c)<\varepsilon$ for some $c \in C\}, \varepsilon>0, C \in C L(X)$, $H(A, B)$

(c) $= \begin{cases}\inf \{\varepsilon>0: A \subset N(\varepsilon, B) \text { and } B \subset N(\varepsilon, A)\}, & \text { if the infimum exists, } \\ \infty, & \text { otherwise, }\end{cases}$ $A, B \in C L(X)$.

The function $H$ is called the generalized Hausdorff distance for $C L(X)$ induced by $d . D(x, A)$ will denote the ordinary distance between $x \in X$ and $A \in C L(X)$.

2. We follow the notation of [2].

$$
\begin{aligned}
& c_{i, k}^{1}=\left\{\begin{array}{ll}
c_{i, k} & \text { for } i \neq k, \\
1-c_{i, k} & \text { for } i=k,
\end{array} \quad i, k=1, \ldots, n,\right. \\
& c_{i, k}^{s+1}= \begin{cases}c_{1,1}^{s} c_{i+1, k+1}^{s}+c_{i+1,1}^{s} c_{i, k+1}^{s} & \text { for } i \neq k, \\
c_{1,1}^{s} c_{i+1, k+1}^{s}-c_{i+1,1}^{s} c_{i, k+1}^{s} & \text { for } i=k,\end{cases} \\
& s=1, \ldots, n-1, i, k=1, \ldots, n-s .
\end{aligned}
$$

The following result is contained in [2].

Lemma. Let $c_{i, k}^{1}>0, i, k=1, \ldots, n$. The system of inequalities

$$
\sum_{k=1}^{n} c_{i, k} r_{k}<r_{i}, \quad i=1, \ldots, n,
$$

has a solution $r_{i}>0, i=1, \ldots, n$, if and only if the following inequalities hold:

Received by the editors January 28, 1975.

AMS (MOS) subject classifications (1970). Primary 54H25.

Key words and phrases. Multivalued mappings, iteration, fixed point theorems.

(1) American Mathematical Society 1976 


$$
c_{i, i}^{s}>0, \quad s=1, \ldots, n, \quad i=1, \ldots, n+1-s .
$$

Suppose that $r_{i}>0, i=1, \ldots, n$, is the solution of the system of inequalities (3). We define

$$
\nu=\max _{i}\left(r_{i}^{-1} \sum_{k=1}^{n} c_{i, k} r_{k}\right) .
$$

In view of the homogeneity of the system of inequalities (3), definition (5) is correct and

$$
0<\nu<1 .
$$

Let $c$ be a real number such that

$$
0 \leqslant c<1-\nu \text {. }
$$

Let $\left(X_{i}, d_{i}\right), i=1, \ldots, n$, be metric spaces. $H_{i}(A, B), i=1, \ldots, n$, will denote the Hausdorff distance between two elements of $C L\left(X_{i}\right), i=$ $1, \ldots, n$, obtained from $d_{i}, i=1, \ldots, n$, and $D_{i}(x, A)$ will denote the ordinary distance between $x \in X_{i}, A \in C L\left(X_{i}\right), i=1, \ldots, n$.

Now we shall prove the following

THEOREM. Let $\left(X_{i}, d_{i}\right), i=1, \ldots, n$, be complete metric spaces and let $a_{i, k} \geqslant 0, b_{i, k} \geqslant 0$ for $i, k=1, \ldots, n$. Let $c_{i, k}=a_{i, k}+b_{i, k}, i, k=1, \ldots, n, b e$ positive and let the numbers $c_{i, k}^{s}, s=1, \ldots, n, i, k=1, \ldots, n+1-s$, defined by (1) and (2) fulfil the inequalities (4). Suppose that the transformations $F_{i}: X_{1} \times \cdots \times X_{n} \rightarrow C L\left(X_{i}\right), i=1, \ldots, n$, fulfil

$$
\begin{gathered}
H_{i}\left[F _ { i } \left(x_{1}, \ldots,\right.\right. \\
\left.\left.x_{n}\right), F_{i}\left(z_{1}, \ldots, z_{n}\right)\right] \leqslant \sum_{k=1}^{n} a_{i, k} d_{k}\left(x_{k}, z_{k}\right) \\
+\sum_{k=1}^{n} b_{i, k} D_{k}\left[x_{k}, F_{k}\left(x_{1}, \ldots, x_{n}\right)\right] \\
+c D_{i}\left[z_{i}, F_{i}\left(z_{1}, \ldots, z_{n}\right)\right]
\end{gathered}
$$

for all $x_{j}, z_{j} \in X_{j}, i, j=1, \ldots, n$, where $c$ fulfils (7). Then the system $\left(F_{1}, \ldots, F_{n}\right)$ has a fixed point, i.e. there exist points $u_{i} \in X_{i}, i=1, \ldots, n$, such that $u_{i} \in F_{i}\left(u_{1}, \ldots, u_{n}\right)$ for all $i=1, \ldots, n$.

Proof. Let $x_{i}^{0} \in X_{i}, i=1, \ldots, n$, and choose $x_{i}^{1} \in F_{i}\left(x_{1}^{0}, \ldots, x_{n}^{0}\right), i$ $=1, \ldots, n$. From (1), (2), (4), the Lemma and (5) we may choose a system of positive numbers $r_{1}, \ldots, r_{n}$ such that

$$
\sum_{k=1}^{n} c_{i, k} r_{k} \leqslant \nu r_{i}, \quad i=1, \ldots, n .
$$

We may assume (from the homogeneity of the above system) that

$$
d_{i}\left(x_{i}^{0}, x_{i}^{1}\right) \leqslant r_{i} \text { and } r_{i} \geqslant 1 \text { for } i=1, \ldots, n .
$$

Let $A, B \in C L\left(X_{i}\right)$ and let $a \in A$. By definition, if $q>0$, then there exists $b \in B$ such that $d_{i}(a, b) \leqslant H_{i}(A, B)+q$. Hence in view of conditions $F_{i}\left(x_{1}^{0}, \ldots, x_{n}^{0}\right), F_{i}\left(x_{1}^{1}, \ldots, x_{n}^{1}\right) \in C L\left(X_{i}\right)$ and $x_{i}^{1} \in F_{i}\left(x_{1}^{0}, \ldots, x_{n}^{0}\right), i$ $=1, \ldots, n$, there exist points $x_{i}^{2} \in F_{i}\left(x_{1}^{1}, \ldots, x_{n}^{1}\right), i=1, \ldots, n$, such that 


$$
d_{i}\left(x_{i}^{1}, x_{i}^{2}\right) \leqslant H_{i}\left[F_{i}\left(x_{1}^{0}, \ldots, x_{n}^{0}\right), F_{i}\left(x_{1}^{1}, \ldots, x_{n}^{1}\right)\right]+\nu .
$$

By induction, we obtain the sequences $\left\{x_{i}^{k}\right\}_{k=1}^{\infty}, i=1, \ldots, n$, of points of $X_{i}$, $i=1, \ldots, n$, such that $x_{i}^{k} \in F_{i}\left(x_{1}^{k-1}, \ldots, x_{n}^{k-1}\right), i=1, \ldots, n, k=1$, $2, \ldots$, and

$$
\begin{aligned}
d_{i}\left(x_{i}^{k}, x_{i}^{k+1}\right) \leqslant & H_{i}\left[F_{i}\left(x_{i}^{k-1}, \ldots, x_{n}^{k-1}\right), F_{i}\left(x_{1}^{k}, \ldots, x_{n}^{k}\right)\right] \\
& +\frac{\nu^{k}}{(1-c)^{k-1}}, \quad i=1, \ldots, n, \quad k=1,2, \ldots .
\end{aligned}
$$

From (11), (8), (10) and (9) we obtain

$$
\begin{aligned}
d_{i}\left(x_{i}^{1}, x_{i}^{2}\right) \leqslant & \sum_{k=1}^{n} a_{i, k} d_{k}\left(x_{k}^{0}, x_{k}^{1}\right)+\sum_{k=1}^{n} b_{i, k} D_{k}\left[x_{k}^{0}, F_{k}\left(x_{1}^{0}, \ldots, x_{n}^{0}\right)\right] \\
& +c D_{i}\left[x_{i}^{1}, F_{i}\left(x_{1}^{1}, \ldots, x_{n}^{1}\right)\right]+\nu \\
\leqslant & \sum_{k=1}^{n}\left(a_{i, k}+b_{i, k}\right) d_{k}\left(x_{k}^{0}, x_{k}^{1}\right)+c d_{i}\left(x_{i}^{1}, x_{i}^{2}\right)+\nu \\
\leqslant & \sum_{k=1}^{n} c_{i, k} r_{k}+c d_{i}\left(x_{i}^{1}, x_{i}^{2}\right)+\nu \\
\leqslant & \nu r_{i}+c d_{i}\left(x_{i}^{1}, x_{i}^{2}\right)+\nu .
\end{aligned}
$$

Thus

$$
d_{i}\left(x_{i}^{1}, x_{i}^{2}\right) \leqslant \frac{\nu}{1-c} r_{i}+\frac{\nu}{1-c} \leqslant 2 \frac{\nu}{1-c} r_{i} .
$$

Recalling (12), (8), (10), (9) and the induction principle, we obtain

$$
d_{i}\left(x_{i}^{k}, x_{i}^{k+1}\right) \leqslant(k+1)(\nu /(1-c))^{k} r_{i}, \quad i=1, \ldots, n, \quad k=1,2, \cdots \text {. }
$$

Now we have

$$
\begin{aligned}
d_{i}\left(x_{i}^{k}, x_{i}^{k+m}\right) & \leqslant d_{i}\left(x_{i}^{k}, x_{i}^{k+1}\right)+\cdots+d_{i}\left(x_{i}^{k+m-1}, x_{i}^{k+m}\right) \\
& \leqslant(k+1)\left(\frac{\nu}{1-c}\right)^{k} r_{i}+\cdots+(k+m)\left(\frac{\nu}{1-c}\right)^{k+m-1} r_{i} \\
& \leqslant \sum_{s=k}^{\infty}(s+1) \alpha^{s} r_{i} \leqslant(k+2) \alpha^{k}(1-\alpha)^{-2} r_{i}
\end{aligned}
$$

for all $k, m \geqslant 1, i=1, \ldots, n$, where $\alpha=\nu /(1-c)$. Thus $\left\{x_{i}^{k}\right\}_{k=1}^{\infty}, i$ $=1, \ldots, n$, are Cauchy sequences and, therefore, $x_{i}^{k} \rightarrow u_{i} \in X_{i}, i$ $=1, \ldots, n$. We claim that $\left(u_{1}, \ldots, u_{n}\right)$ is a fixed point of $\left(F_{1}, \ldots, F_{n}\right)$. Actually

$$
\begin{aligned}
& D_{i}\left[u_{i}, F_{i}\left(u_{1}, \ldots, u_{n}\right)\right] \leqslant d_{i}\left(u_{i}, x_{i}^{k+1}\right)+D_{i}\left[x_{i}^{k+1}, F_{i}\left(u_{1}, \ldots, u_{n}\right)\right] \\
& \quad \leqslant d_{i}\left(u_{i}, x_{i}^{k+1}\right)+H_{i}\left[F_{i}\left(x_{1}^{k}, \ldots, x_{n}^{k}\right), F_{i}\left(u_{1}, \ldots, u_{n}\right)\right] \\
& \quad \leqslant d_{i}\left(u_{i}, x_{i}^{k+1}\right)+\sum_{s=1}^{n} a_{i, s} d_{s}\left(x_{s}^{k}, u_{s}\right)
\end{aligned}
$$




$$
\begin{aligned}
& \quad+\sum_{s=1}^{n} b_{i, s} D_{s}\left[x_{s}^{k}, F_{s}\left(x_{1}^{k}, \ldots, x_{n}^{k}\right)\right]+c D_{i}\left[u_{i}, F_{i}\left(u_{1}, \ldots, u_{n}\right)\right] \\
& \leqslant d_{i}\left(u_{i}, x_{i}^{k+1}\right)+\sum_{s=1}^{n} a_{i, s} d_{s}\left(x_{s}^{k}, u_{s}\right) \\
& \quad+\sum_{s=1}^{n} b_{i, s} d_{s}\left(x_{s}^{k}, x_{s}^{k+1}\right)+c D_{i}\left[u_{i}, F_{i}\left(u_{1}, \ldots, u_{n}\right)\right]
\end{aligned}
$$

Hence,

$$
\begin{aligned}
& D_{i}\left[u_{i}, F_{i}\left(u_{1}, \ldots, u_{n}\right)\right] \\
& \leqslant \frac{1}{1-c}\left[d_{i}\left(u_{i}, x_{i}^{k+1}\right)+\sum_{s=1}^{n} a_{i, s} d_{s}\left(x_{s}^{k}, u_{s}\right)\right. \\
& \left.\quad+\sum_{s=1}^{n} b_{i, s} d_{s}\left(x_{s}^{k}, x_{s}^{k+1}\right)\right] \rightarrow 0 \quad \text { as } k \rightarrow \infty .
\end{aligned}
$$

Since $F_{i}\left(u_{1}, \ldots, u_{n}\right)$ is closed, this means that $u_{i} \in F_{i}\left(u_{1}, \ldots, u_{n}\right), i$ $=1, \ldots, n$, which completes the proof.

\section{REFERENCES}

1. S. Czerwik, Fixed point theorems for system multi-valued mappings, Coll. Math. (to appear).

2. J. Matkowski, Some inequalities and a generalization of Banach's principle, Bull. Acad. Polon. Sci. Sér. Sci. Math. Astronom. Phys. 21(1973), 323-324. MR47 \#5664.

3. S. B. Nadler, Jr., Multi-valued contraction mappings, Pacific J. Math. 30(1969), 475-488. MR40 \#8035.

4. - Some results on multi-valued contraction mappings, Set-Valued Mappings, Selections and Topological Properties of $2^{x}$ (Proc. Conf., SUNY, Buffalo, N. Y., 1969), Lecture Notes in Math., vol. 171, Springer-Verlag, Berlin and New York, 1970, pp. 6469. MR43 \# 1148.

5. S. Reich, Kannan's fixed point theorem, Boll. Un. Mat. Ital. 4(1971), 1-11. MR46 \#4293.

Institute of Mathematics, Silesian University, 40-007 Katowice, Poland 